\title{
Field Potential Signature of Distinct Multicellular Activity Patterns in the Mouse Hippocampus
}

\author{
Susanne Reichinnek, ${ }^{1,2}$ Thomas Künsting, ${ }^{1}$ Andreas Draguhn, ${ }^{1,2}$ and Martin Both ${ }^{1,2}$ \\ ${ }^{1}$ Institute of Physiology and Pathophysiology, and 'Interdisciplinary Center for Neurosciences, University of Heidelberg, D-69120 Heidelberg, Germany
}

Cognitive functions go along with complex patterns of distributed activity in neuronal networks, thereby forming assemblies of selected neurons. To support memory processes, such assemblies have to be stabilized and reactivated in a highly reproducible way. The rodent hippocampus provides a well studied model system for network mechanisms underlying spatial memory formation. Assemblies of place-encoding cells are repeatedly activated during sleep-associated network states called sharp wave-ripple complexes (SPW-Rs). Behavioral studies suggest that at any time the hippocampus harbors a limited number of different assemblies that are transiently stabilized for memory consolidation. We hypothesized that the corresponding field potentials (sharp wave-ripple complexes) contain a specific signature of the underlying neuronal activity patterns. Hence, they should fall into a limited number of different waveforms. Application of unbiased sorting algorithms to sharp wave-ripple complexes in mouse hippocampal slices did indeed reveal the reliable recurrence of defined waveforms that were robust over prolonged recording periods. Single-unit discharges tended to fire selectively with certain SPW-R classes and were coupled above chance level. Thus, field SPW-Rs of different waveforms are directly related to the underlying multicellular activity patterns that recur with high fidelity. This direct relationship between the coordinated activity of distinct groups of neurons and macroscopic electrographic signals may be important for cognition-related physiological studies in humans and behaving animals.

\section{Introduction}

The mammalian hippocampus displays a variety of statedependent network oscillations (O'Keefe and Nadel, 1978; Buzsáki and Chrobak, 1995; Klausberger and Somogyi, 2008) that entrain principal cells into coactive neuronal assemblies (Buzsáki and Draguhn, 2004). A well studied behavioral correlate of these neuronal activation patterns is spatial navigation. During exploration of a new environment, pyramidal cells acquire different place-selective firing properties (O'Keefe and Dostrovsky, 1971). By systematic changes of their temporal relationship to the underlying theta network oscillation, they form sequential activation patterns that can represent the animal's trajectory through space (O'Keefe and Recce, 1993). These sequences are replayed, at faster pace, during subsequent sleep or inactive wakefulness (Wilson and McNaughton, 1994; Nadasdy et al., 1999; Foster and Wilson, 2006). Reactivation occurs on top of brief field potential transients called sharp wave-ripple events (SPW-Rs) that propagate along the hippocampal loop toward the entorhinal cortex (Chrobak and Buzsáki, 1996; Bragin et al., 1999). Oscillation-associated hippocampal activity patterns may therefore form an important example for the formation and stabilization of representations by neuronal assemblies (Hebb, 1949).

\footnotetext{
Received May 18, 2010; revised July 30, 2010; accepted Sept. 14, 2010.

This work was supported by Sonderforschungsbereich (SFB) 488 and SFB 636. We thank Dr. A. Ponomarenko, C. Fiebach, D. Durstewitz, and R. Traub for helpful discussions and technical support.

Correspondence should be addressed to Martin Both at the above address. E-mail: mboth@ physiologie.uni-heidelberg.de.

D0I:10.1523/JNEUROSCI.2535-10.2010

Copyright $\odot 2010$ the authors $\quad 0270-6474 / 10 / 3015441-09 \$ 15.00 / 0$
}

Stable patterns of place cell activity form within minutes after exposure to a new spatial context (Wilson and McNaughton, 1993; Frank et al., 2004). They are maintained over several days and can be rapidly reactivated on repeated exposure to the same environment (Leutgeb et al., 2005). As a consequence, multiple distinct assemblies should be present in the hippocampus at any time, consistent with model calculations of hippocampal memory capacity (Lisman, 1999; Leibold and Kempter, 2006). Although recordings from multiple units confirm the general concept, they only reflect a small minority of cells within each assembly. An alternative approach may be the recording of field potentials that comprise a weighted average of multiple subthreshold synaptic potentials, action potentials, and electrical coupling. Previous work has shown that, in principle, defined field potential waveforms can be reproducibly generated by repetitive activation of a similar set of neurons (Buzsáki, 1989). In this work, large-amplitude, short-duration spikes were evoked in the hippocampus of fimbria-fornix-lesioned rats. Repetitive stimulation at high frequency stabilized the activated network such that events with the same shape occurred spontaneously afterward. In a more physiological paradigm, Csicsvari et al. (2000) could relate the firing of different units in CA3 to the subsequent occurrence of sharp wave-ripple events in more proximal or distal portions of CA1. Therefore, it may be feasible to detect differences in the electrographic signature of different assemblies by extracellular recordings. If these extracellularly recorded potentials would reflect individual features of the underlying assemblies, they would provide an easy accessible correlate of different memory representations in the CNS. 
Sharp wave-ripple complexes are ideally suited to test this hypothesis: (1) they are spatially and temporally well delineated, allowing for the collection of numerous distinct field events; (2) they go along with the repetitive activation of multiple different, but specific patterns of spikes in selected place cells; (3) they provide a good signal-to-background ratio (i.e., most pyramidal cells are inhibited during SPW-R, leaving only participating cells active). We therefore recorded spontaneously occurring sharp wave-ripple complexes in mouse hippocampal slices in vitro (Maier et al., 2002, 2003). Unbiased sorting of different waveforms revealed multiple different types of SPW-Rs that recurred reliably over prolonged recording periods. Moreover, discharges of single neurons were coupled to a specific subset of field SPW-R patterns. These findings show that averaged signals from small networks can be used to identify specific and individual multicellular activity patterns.

\section{Materials and Methods}

Experiments were performed on male C57BL/6 mice of 4-8 weeks age and were approved by the state government of BadenWürttemberg. Mice were anesthetized with ether and decapitated before the brain was removed and transferred into cooled artificial CSF (ACSF) (1-4 ${ }^{\circ} \mathrm{C}$ ), containing the following (in $\mathrm{mM}$ ): 124 $\mathrm{NaCl}, 3.0 \mathrm{KCl}, 1.8 \mathrm{MgSO}_{4}, 1.6 \mathrm{CaCl}_{2}, 10$ glucose, $1.25 \mathrm{NaH}_{2} \mathrm{PO}_{4}, 26 \mathrm{NaHCO}_{3}$, saturated with $95 \%$ $\mathrm{O}_{2} / 5 \% \mathrm{CO}_{2}, \mathrm{pH} 7.4$ at $37^{\circ} \mathrm{C}$. After removal of the cerebellum and frontal brain structures, horizontal slices of $450 \mu \mathrm{m}$ were cut using a Leica Vibratome (VT1000 S). Slices were transferred to a Haas-type interface recording chamber at $34 \pm$ $1^{\circ} \mathrm{C}$ and were allowed to recover for at least $2 \mathrm{~h}$.

Recordings. Field potentials and unit discharges were recorded with two to three tetrodes that were placed at and then moved slightly into the surface of the slice in CA1 or CA3 stratum pyramidale. The tetrodes were made of four twisted $12.5-\mu \mathrm{m}$-diameter tungsten wires (California Fine Wire) and were connected to separate DPA-2FX amplifiers (npi electronics). Signals were amplified $100 \times$, low-pass filtered at $10 \mathrm{kHz}$, high-pass filtered at $0.3 \mathrm{~Hz}$, and digitized at $20 \mathrm{kHz}$ for off-line analysis (1401 interface and Spike-2 data acquisition program; CED).

Field potentials in stratum radiatum of CA1 were recorded with ACSFfilled glass microelectrodes (tip diameter, $2-4 \mu \mathrm{m}$ ). Voltage signals were amplified $100 \times$, low-pass filtered at $2 \mathrm{kHz}$, high-pass filtered at $0.3 \mathrm{~Hz}$ (EXT10-2FX amplifiers; npi electronics), and digitized at $20 \mathrm{kHz}$. To elicit SPW-R-like activity in CA1, weak electrical stimulation (100 $\mu \mathrm{s}$ square voltage pulses) was applied in CA3b and CA3c with bipolar platinum/iridium wires (Science Products; $100 \mathrm{k} \Omega$ at $1 \mathrm{kHz} ; 75 \mu \mathrm{m}$ tip distance). The constant excitability of slices was tested by electrical stimulation of the Schaffer collaterals. Slices that varied $>30 \%$ were excluded from the analysis. To avoid shifts in waveform composition attributable to deteriorating recording conditions, we additionally required stability of mean SPW-R frequency and amplitude within 10\% during the experiment

Analysis of field potentials. Signals were sampled with the Spike- 2 program (CED) and analyzed off-line using custom routines written in Matlab (The
MathWorks). Sharp waves were detected from low-pass-filtered raw data $(50 \mathrm{~Hz})$ as local maxima with amplitudes $>0.15-0.2 \mathrm{mV}$ within $30 \mathrm{~ms}$ time windows. This value corresponds to 4 SDs of event-free baseline noise (Both et al., 2008) yielding stable and reliable detection of SPW-Rs (as confirmed by visual inspection of traces and detected events). Subsequently, SPW-R complexes were analyzed with continuous wavelet transform (complex Morlet wavelet) (Both et al., 2008), starting $33 \mathrm{~ms}$ before and ending $67 \mathrm{~ms}$ after the peak of the detected sharp wave. From this wavelet spectrogram (50-300 Hz divided into 81 bins on a log scale), we extracted the time delay of the maximum ripple oscillation with respect to the underlying sharp wave, the leading ripple frequency, and the peak power of the oscillation at frequencies $>140 \mathrm{~Hz}$. The same parameters were evaluated for electrically evoked SPW-Rs after removal of the stimulus artifact. SPW-R amplitude and frequency were calculated from $5 \mathrm{~min}(300 \mathrm{~s})$ bins.

Self-organizing maps. SPW-R waveforms were classified using selforganizing maps (SOMs) (Kohonen, 1995). SOMs are an unsupervised learning algorithm that provides a projection of high-dimensional data onto a two-dimensional classification map that preserves some of the neighborhood relationship or topography of the original data. After the training phase, each SPW-R can be associated with (and thereby classified by) a map unit that is closest to it in the representing vector space [the BMMU (best matching map unit); please do not confuse these with 

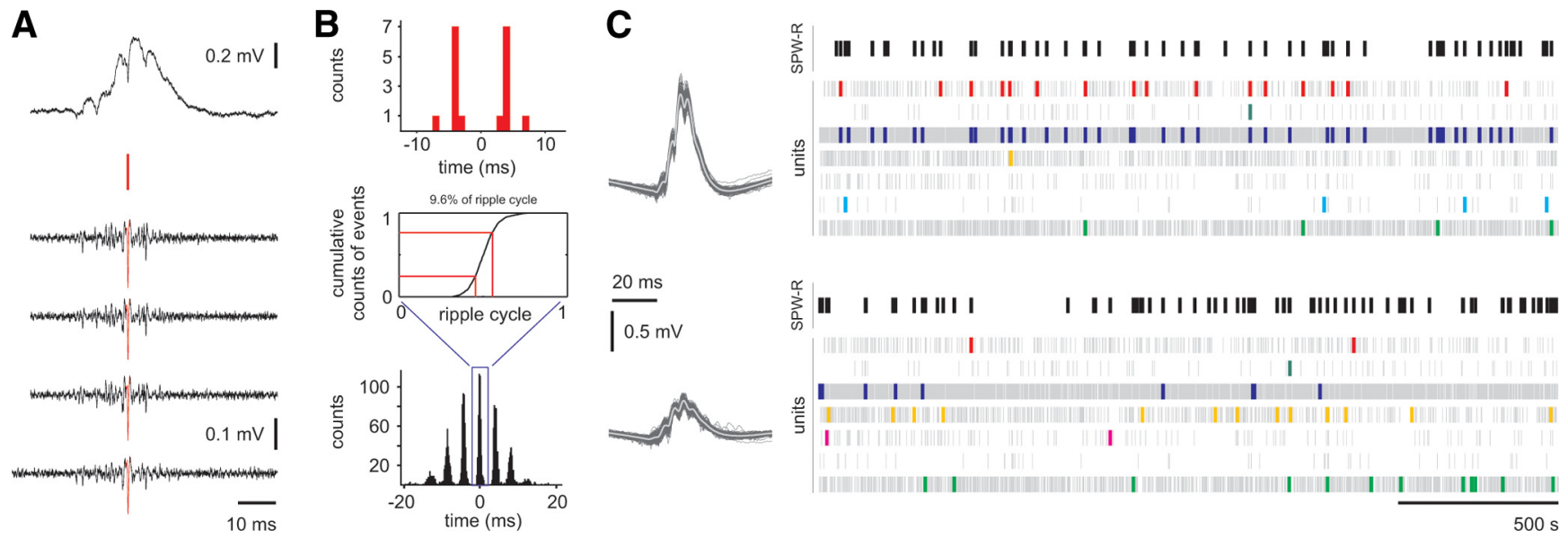

Figure 2. Unit discharges in CA1 show preferences to certain SPW-R waveforms. $A$, Local field potential samples of SPW- $R$ (top traces) and high-pass-filtered tetrode recordings (four bottom traces). The red ticks and highlighted data traces indicate the firing of one putative pyramidal neuron. $\boldsymbol{B}$, Autocorrelation (top panel), coupling precision to the ripple cycle (middle panel), event cross-correlation and ripple troughs (bottom panel) for the unit shown in $A$. Note high precision of coupling to field ripples. C, Unit discharges (right panel, colored ticks) during the occurrence of two distinct SPW-R waveforms (left panel). The gray unit ticks indicate times when the unit was active during other SPW-R shapes. Note the specificity of units to different SPW-R waveforms.

"units" from electrophysiological measurements]. We chose this approach as one cannot exclude that there are several problems associated with the classification of local field potentials. First, there might not be a clustering tendency in the data set at all but rather a continuum of different shapes. Second, shapes from the activity of possibly stable neuronal groups might change over time as other parameters (e.g., volume conductance attributable to activity-dependent changes in extracellular ion concentrations) might change. Third, the exact composition of neuronal ensembles might fluctuate and/or change systematically over the course of the experiment. Moreover, different neuronal assemblies might, by chance, generate the same field potential shape and are therefore classified into the same cluster. Certainly the use of SOMs for classifying field potential shapes does not circumvent these problems, but provides additional means to interpret the resulting classification in excess of simply evaluating the resulting class membership: similar field potential shapes are classified next to each other on the SOM. If a distinct assembly is reactivated several times and the corresponding field potential shapes are similar to each other, it will at least be classified to a connected and circumscribed area on the SOM. Similarly, a neuron that is a member of a distinct assembly will show a characteristic distribution toward connected field potential shapes on the SOM.

For details about the exact implementation of SOMs, supplemental Materials and Methods (available at www.jneurosci.org as supplemental material) provides information describing the classification and comparison of SPW-R waveforms by SOMs.

Unit analysis. To detect extracellularly recorded action potentials (units), raw data were high-pass filtered at $500 \mathrm{~Hz}$ and upsampled from 20 to $100 \mathrm{kHz}$ with a Nyquist algorithm (Blanche and Swindale, 2006). Single events were extracted by setting a negative threshold at 4.5 SDs from background noise. Subsequently, putative units were analyzed by principal component analysis (PCA) and clustered using the first three principal components of each tetrode channel in the open source program Klustakwik (Harris et al., 2000). Unit clusters were finally identified after visual inspection with the open source program Klusters (Hazan et al., 2006), using only event waveforms with clear separation from other units (see supplemental Fig. S5, available at www.jneurosci.org as supplemental material). Our approach to link cellular activity with the local field potential does not require that the unit clusters contain only spikes emitted by a single neuron. In fact, several of the detected events consisted of a sequence of individual events (see waveform; supplemental Fig. S5A, unit 7, available at www.jneurosci.org as supplemental material). These events can be seen as recurring distinct neuronal sequences and further substantiate the relationship between cellular firing and field events. Therefore, reasonable application of our algorithms solely requires a stable unit waveform that is well isolated across the recording period to examine neuronal activity patterns rather than single cells. Unfortunately, events consisting of multiple spikes do not match well into the parameter space (principal components) used for clustering and often contain few spikes. Therefore, these clusters tend to scatter and have low-quality measures. Meaningful classification for all of our clusters with low-quality measures and clusters that did not meet stability was anew confirmed by visual inspection and distinctness concerning high-pass-filtered raw data waveforms. Resulting clusters quality measures are $L_{\text {ratio }}$ of 0.78 [median; 25th percentile $\left(\mathrm{P}_{25}\right), 0.35 ; 75$ th percentile $\left.\left(\mathrm{P}_{75}\right), 2.4\right]$ and isolation distance of 15.4 (median; $\mathrm{P}_{25}, 10.8 ; \mathrm{P}_{75}, 18.6$ ) (Schmitzer-Torbert et al., 2005). For each identified unit, we calculated spike width and amplitude of unit waveform, spiking frequency, and interspike intervals within $300 \mathrm{~s}$ sliding windows as well as the autocorrelation function. Coupling precision of unit firing to field ripples was computed from event cross-correlations of unit discharge times and ripple troughs (Both et al., 2008). Stable entrainment of units by the underlying ripple oscillation is apparent by distinct peaks of the crosscorrelation function at intervals corresponding to the cycle length of ripples (see Fig. $2 \mathrm{~B}$ ). Phase-coupling was quantified from cumulative histograms of spikes with respect to their time of occurrence within a single ripple cycle. Random distribution would yield $50 \%$ of spikes occurring during $50 \%$ of one ripple cycle $(\sim 5 \mathrm{~ms})$. Lower time intervals indicate phase-coupling. The units were not further divided into putative pyramidal cells or interneurons as "classical" parameters from in vivo recordings based on autocorrelation, firing frequency, and spike width cannot directly be applied to units firing preferentially during SPW-R events in hippocampal slices in vitro.

We constructed "unit hit maps" to analyze whether units fire preferentially coupled to certain field potential waveforms. Therefore, SPW-Rs were classified by the SOM, and unit events occurring during waveforms of these SPW-R classes were summed up to construct a histogram map. This map was normalized by the probability of occurrence of each SPW-R class. Specificity of unit firing was quantified as information and sparsity, as defined by Skaggs et al. (1993):

$I($ unit $\mathrm{SPW})=\sum_{i}^{\mathrm{SPW}}\left[p\left(\mathrm{SPW}_{i}\right) * p\left(\right.\right.$ unit $\left.\left.\mathrm{SPW}_{i}\right){ }^{*} \log 2\left(\frac{p\left(\text { unit }^{\mathrm{SPW}} \mathrm{SP}_{i}\right)}{p(\text { unit })}\right)\right]$,

where $p(x \mid y)$ is the conditional probability of $x$ given $y$, and $p(z)$ the marginal probability that $z$ occurs.

Sparsity was calculated according to Treves and Rolls (1994) and Skaggs et al. (1993), with $\lambda$ as total average firing rate and $\lambda_{i}$ as average firing rate during a certain SPW, as follows:

$$
S(\text { unit } \mid \mathrm{SPW})=\left[\frac{\lambda^{2}}{\sum_{i}^{\mathrm{SPW}} p\left(\mathrm{SPW}_{i}\right){ }^{*}{ }_{i}^{2}}\right] .
$$

Random distributions were calculated by shuffling spikes with all sharp waves from the respective experiment (1000 repetitions). Information and 
sparsity of each unit were compared with the resulting random distributions, considering values outside the $95 \%$ confidence interval as significant.

For a more rigid and physiologically more accurate randomization, we additionally used a second randomization. For each unit, we drew randomly as many SPW-Rs as this unit had discharged on SPW-Rs during the recording. In contrast to the conventional randomization, these SPW-Rs were taken only from the pool of SPW-Rs in which we had detected one or more units in the original data trace. By this, we corrected for a potential bias toward SPW-Rs that might have been generated far away from the recording tetrode and lacked detectable and separable unit discharges. Again, we calculated the information and sparsity of 1000 random distributions and compared them with the actual values, considering values outside the $95 \%$ confidence interval as significant. Our data show that individual units discharge on defined subsets of SPW-Rs. Quantifying the information and sparsity of occupancy on the unit hit maps were significantly different from random values for 95 units (i.e., $34 \%$; information) and 84 units (i.e., $30 \%$; sparsity), respectively [difference for both parameters: 78 units (i.e., 28\%)] depicted in one detailed example in which parameters for all units of one representative experiment are shown (supplemental Fig. S6, available at www.jneurosci.org as supplemental material).

Statistics. Quantitative results are given as mean \pm SEM if data was normally distributed or as median with 25 th and 75th percentiles. Significance of results was tested by Wilcoxon's rank sum test or Kruskal-Wallis nonparametric one-way ANOVA, and $p<0.05$ was regarded as significant.

\section{Results}

\section{SPW-R waveforms reoccur over time}

SPW-Rs are spatially and temporally restrained patterns of network activity that are generated in CA3 and propagate along the hippocampal output loop. The spontaneous network events can be recorded as field potential waves in CA1 of living rodents (Chrobak and Buzsáki, 1996) or brain slices (Maier et al., 2003). We asked whether these weighted average signals of neuronal activity contain direct information about the underlying multicellular activity patterns. Recording with two to three tetrodes along the CA1 pyramidal cell layer of mouse hippocampal slices revealed spontaneously occurring SPW-Rs with similar basic properties but strong variability between their individual waveforms (Fig. $1 A, B$ ). On closer inspection, we found that certain SPW-R waveforms occurred repetitively over time, even during prolonged recordings for $\sim 4000 \mathrm{~s}$ (Fig. $1 C, D$ ). This observation was confirmed by averaging similar numbers of randomly chosen SPW-Rs, or SPW-Rs of the same waveform, respectively. Whereas averages of randomly chosen events resulted in smooth field potentials, averages of similarly looking SPW-Rs maintained the fine structure of superimposed high-frequency "ripple" oscillations (Fig. 1D). We conclude that certain SPW-R waveforms recur with high fidelity. Does this reflect the repetitive activation of well defined underlying multicellular activity patterns? To answer this question, we performed a rigorous sorting of SPW-R field events before correlating different waveforms with concomitant discharges single units.

\section{SPW-R waveforms can be sorted by SOMs}

We sorted multiple SPW-R events from individual hippocampal slices $(\sim 10,000$ SPW-Rs from $\sim 4000 \mathrm{~s}$ of raw data in each slice with stable recording conditions; median, 10,072; $\mathrm{P}_{25}, 8262 ; \mathrm{P}_{75}$, 12,$841 ; n=19$ slices). Classification followed two steps: first, we applied PCA (see supporting information, available at www. jneurosci.org as supplemental material) to describe the events by a small set of independent parameters. Subsequently, we sorted the resulting vectors by SOMs (see Materials and Methods) (see supporting information, available at www.jneurosci.org as supplemental material), an automatic unsupervised learning algo-
A
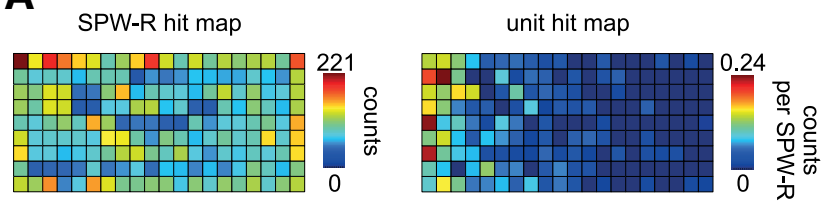

B
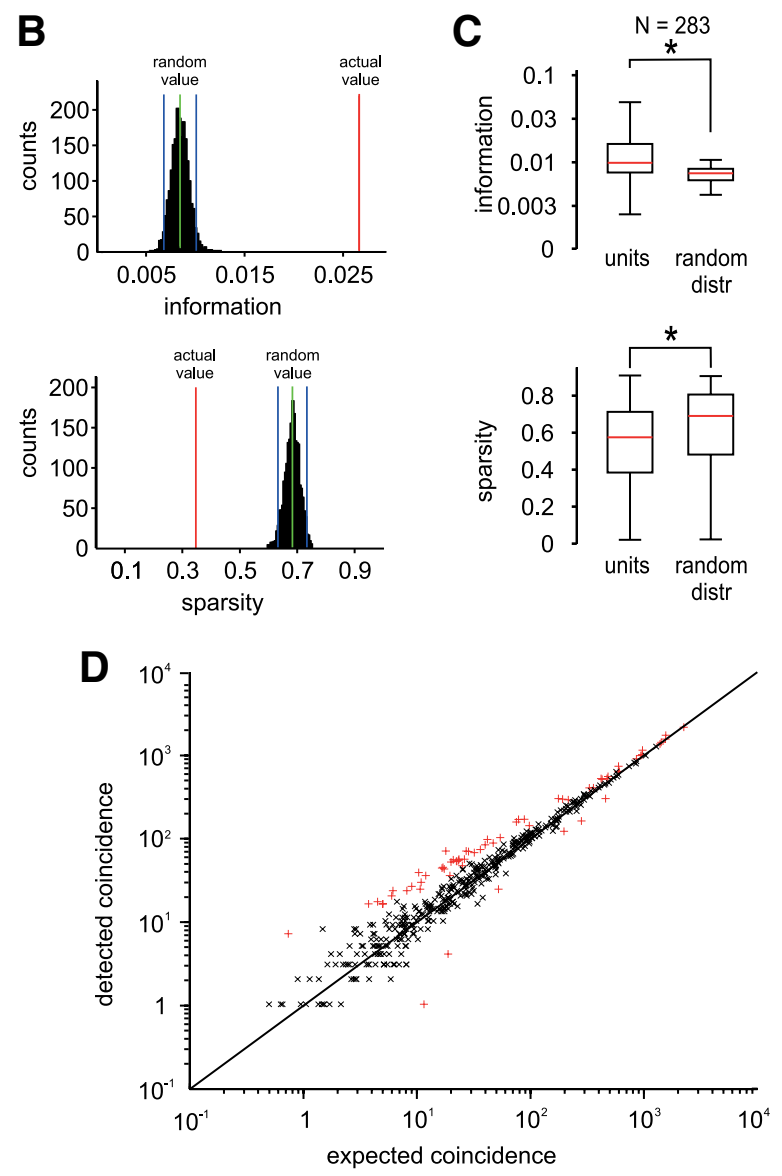

Figure 3. Unit discharges in CA1 show preferences to certain SPW-R waveforms. A, SPW-R hit map (left) and unit hit map (right) for the unit shown in $\boldsymbol{A}$ and $\boldsymbol{B}$. This unit discharges only on specific SPW-R waveforms, belonging to a distinct area of the SPW-R hit map. $\boldsymbol{B}$, Information (top panel) and sparsity (bottom panel) for the unit shown in $\boldsymbol{A}$ and $\boldsymbol{B}$ reveal a significant difference between measured values and a random distribution based on unit firing frequency. C, Specificity of 283 SPW-R coupled units shows significant differences between measured and random values for information (top panel) and sparsity (bottom panel) revealing a specific coupling of units to certain SPW-R waveforms. $D$, Units in CA1 form multicellular activity patterns and show coupled discharge during SPW-R. Fourteen percent $(n=65)$ of all unit pairings ( $n=461$ ) show coincidences that are significantly different form the value expected from their individual firing rates (red plus signs, $p<0.05$, Fisher's exact test with Bonferroni's correction); ${ }^{*} p<0.05$.

rithm (Kohonen, 1995). In short, a rectangular map of prototypic waveforms is formed by an iterative training phase and optimized to represent and classify the data as a whole. The resulting twodimensional representation maintains a topographic order of the prototypic waveforms [i.e., "similar" SPW-Rs fall onto closely located SOM areas (so-called "map units")] (for details, see supporting information, available at www.jneurosci.org as supplemental material). Correct sorting of waveforms and similaritybased topography of the map was confirmed by averaging individual events from the same SOM unit (supplemental Fig. S1, available at www.jneurosci.org as supplemental material). Together, these findings are compatible with the stable expression 

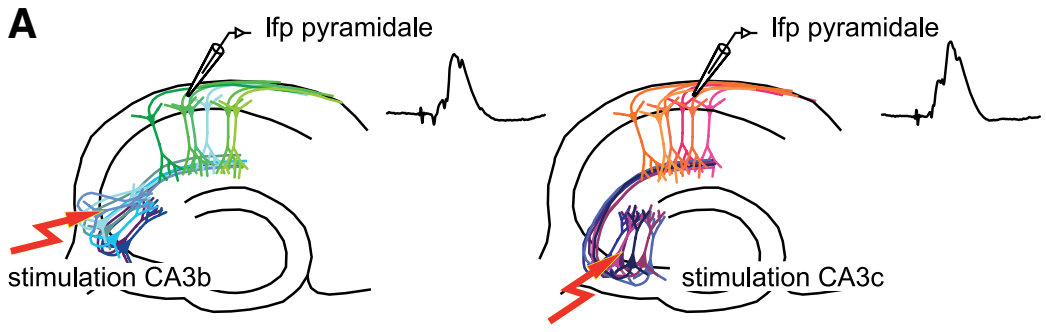

B
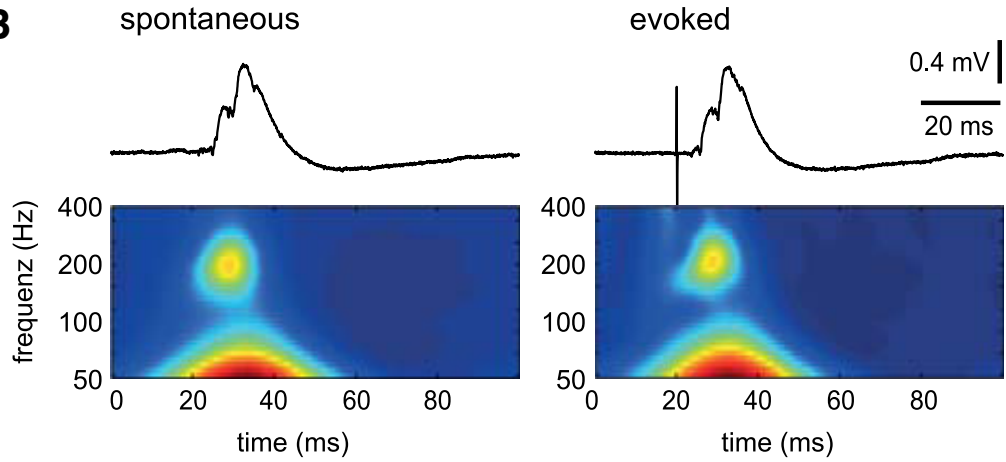

C
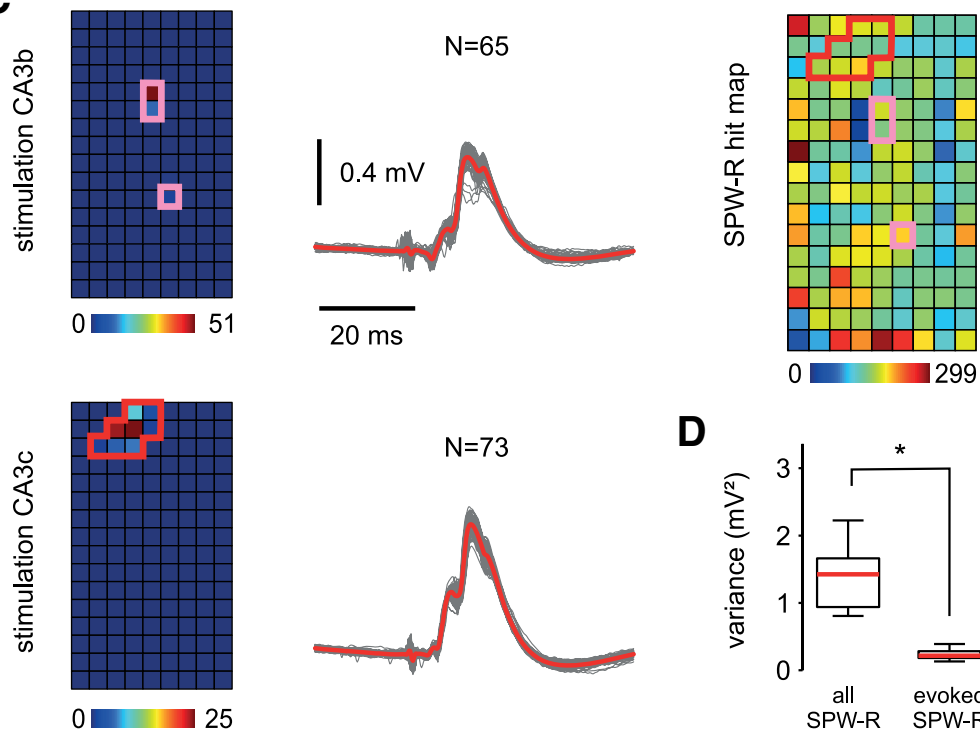

D

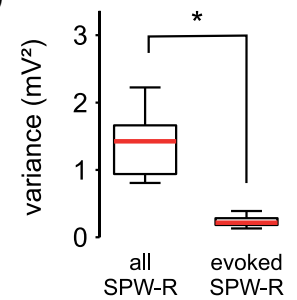

Figure 4. Weak electrical stimuli in CA3 activate specific patterns in CA1. A, Activation of distinct neuronal subpopulations in CA3 activates different upstream neuronal cells resulting in different SPW-R waveforms. $\boldsymbol{B}$, Comparison between spontaneous and evoked SPW-Rs reveals no difference in waveform and frequency components (for details, see supplemental Fig. S9, available at www.jneurosci.org as supplemental material). C, Weak activation of CA3 cells at 300 ms after a strong stimulation. Evoked SPW-Rs are confined to few, strongly connected areas within the SOM (left panel) created of spontaneous and evoked events. Furthermore, evoked SPW-Rs show clearly distinct, but reproducible stable waveforms at two different stimulation locations (middle). On a SPW-R hit map generated from spontaneous events (right panel), these elicited SPW-R events are homogenously distributed. $D$, The variance of evoked SPW-R events is significantly smaller than the variance of all SPW-Rs, indicating a specific interaction between stimulated cells in CA3 and resulting activated cells in CA1; ${ }^{*} p<0.05$.

and recurrent activation of a limited set of different SPW-R field potentials.

\section{Units fire preferentially on defined SPW waveforms}

Action potentials of CA1 pyramidal cells occur at precisely defined times during SPW-R (Ylinen et al., 1995; Csicsvari et al., 2000). Assuming that different SPW-R waveforms reflect different underlying multicellular activity patterns, individual neurons should preferentially fire on a defined subset of SPW-Rs. This should result in a nonrandom distribution of unit discharges on the SOM of field SPW-Rs.

Using three tetrodes along the CA1 pyramidal band, we identified 283 SPW-Rcorrelated units in 21 slices (19 animals) by their discrete spike waveform (Fig. $2 A)$. Discharge frequency was $0.07 \mathrm{~Hz}$ (median; $\mathrm{P}_{25}, 0.02 \mathrm{~Hz} ; \mathrm{P}_{75}, 0.18 \mathrm{~Hz}$ ). Similar to SPW-R in vivo (Csicsvari et al., 1999), discharge probability increased strongly during SPW-R events $(9.9 \pm 1.9$ fold). Individual units fired on $2.6 \%$ of the spontaneously occurring SPW-Rs (median; $\left.\mathrm{P}_{25}, 1.0 \% ; \mathrm{P}_{75}, 6.6 \%\right)$. In addition, units were entrained by the superimposed ripple oscillations (Csicsvari et al., 1999; Both et al., 2008). Coupling precision was $0.99 \pm 0.03 \mathrm{~ms}$, corresponding to $22 \pm$ $1 \%$ of a single ripple cycle. Most units (236 of 283; 83\%) had a coupling precision $<35 \%$ (for an example, see Fig. $2 B$; chance value is $50 \%$, and lower values indicate higher precision).

We then asked whether discharges of identified units occurred randomly on all possible SPW-R waveforms or, alternatively, on a defined subset of SPW-R events (for an example, see Fig. 2C). Approximately one-half of the unit hit maps showed a nonrandom distribution (Fig. $3 A$; supplemental Fig. S6, available at www.jneurosci.org as supplemental material), indicating that individual neurons fire preferentially on a subset of network events. To quantify this specificity, we computed information and sparsity for each unit and compared the values to random distributions from shuffled data (Fig. 3B; supplemental Fig. S6, available at www.jneurosci.org as supplemental material) (see Materials and Methods) (see supplemental information, available at www.jneurosci.org as supplemental material) (Brun et al., 2008). This test revealed significant deviations from random values for both parameters in 115 of 283 (41\%) units (Fig. 3C). Information varied significantly from chance in 138 units (49\%) and sparsity was nonrandom in 126 units (45\%). A more rigid randomization that excluded contributions from remote SPW-R events did still reveal significant specificity in approximately one-third of all units (see Materials and Methods). As this specificity could be attributable to a rather trivial "preference" of unit entrainment by large amplitude SPW-Rs, we performed the following tests: first, we identified several units with strong preference to smallamplitude SPW-Rs (for two examples, see supplemental Fig. S6 B, F, available at www.jneurosci.org as supplemental material). Second, we estimated the amplitude bias of units by computing the median amplitude of the SPW-Rs on which the individual units fired. The distribution is slightly biased toward large amplitudes but shows clearly that many units are distributed about median or small SPW-Rs (see supplemental Fig. S6 $H$, available at www.jneurosci.org as supplemental material). Third, we reclassified the SPW-R wave- 
forms after normalizing all events by their amplitudes. This rigorous test resulted in similar percentages of units with waveform selective firing (38\% using both criteria, $42 \%$ for sparsity, and $48 \%$ for information). Thus, SPW-R waveform selectivity of unit discharges is not an artifact of amplitude selectivity.

These data show that individual units discharge on defined subsets of SPW-Rs. In consequence, different units recorded from the same slice may be coherently activated by the same events, forming part of a strongly connected neuronal group. We tested this in slices in which multiple clearly distinct units could be identified ( 21 slices; $3-22$ units per slice). If two cells are firing independently during SPW-Rs with probabilities $p(x)$ and $p(y)$, coincident discharges during a given SPW-R should be observed with the probability $p(x) \cdot p(y)$. We computed this value for units recorded at the two most distant tetrodes and compared it with our experimental results (Fisher's exact test with Bonferroni's correction). Coupled discharges were significantly above chance in 65 of 461 unit pairs $(14.1 \%$; $p<0.05)$ (Fig. 3D). This finding shows that different neurons are indeed coupled to common sets of SPW-R waveforms, constituting a direct observation of stable multicellular activity patterns in CA1.

\section{Activation of neuronal subpopulations induces distinct SPW-R waveforms}

SPW-Rs are generated in the hippocampal subfield CA3 and propagate along CA1 toward the subiculum and entorhinal cortex. Therefore, different assemblies in CA1 may be activated by different upstream assemblies in CA3 (Fig. 4A). Indeed, weak electrical stimulation at the border between stratum pyramidale and stratum radiatum of CA3b or CA3c elicited typical SPW-Rs in CA1 (Both et al., 2008) (17 slices from eight animals) (Fig. $4 B$; supplemental Fig. S4, supporting information, available at www. jneurosci.org as supplemental material).

Stimulation at random time points during ongoing spontaneous SPW-R activity resulted in variable events (supplemental Fig. S4, available at www.jneurosci.org as supplemental material). However, a strong "reset" stimulus $300 \mathrm{~ms}$ before the evoked SPW-R (supplemental Fig. S4, available at www.jneurosci.org as supplemental material) induced a brief pause in spontaneous network activity and allowed for the generation of very stable SPW-R waveforms. This was reflected by a significant reduction in waveform variance [median: random pulse, $0.75 \mathrm{mV}^{2}(n=$ $15)$; prepulse, $0.22 \mathrm{mV}^{2}(n=8), p<0.05$, Mann-Whitney] (supplemental Fig. S4, available at www.jneurosci.org as supplemental material). Supportively, waveforms of evoked SPW-Rs had lower variance than spontaneously occurring SPW-Rs $(n=$ 8; median of variance for all SPW-Rs, $1.43 \mathrm{mV}^{2}$; median of evoked SPW-Rs, $0.22 \mathrm{mV}^{2}$; $p<0.05$, Mann-Whitney) (Fig. 4D), indicating a highly specific input of CA3 to CA1.

Stimulus-evoked SPW-R complexes appeared highly similar to certain waveforms of spontaneous events in the same slice (Fig. $4 B$ ), and both types of events shared the same map units (Fig. $4 C$ ). Most importantly, stimulation-evoked SPW-Rs (with prepulse conditioning) consistently mapped onto restricted sections of the respective SOM, covering $\sim 11 \%$ of the full SOM (median; $\left.\mathrm{P}_{25}, 3 \% ; \mathrm{P}_{75}, 14 \% ; n=8\right)$. This limited occupancy of the reference SOM poses a lower limit of different SPW-R types in the order of 10. It also indicates that well defined subnetworks in CA1 can be reproducibly activated by activation of small, spatially segregated networks within CA3.

As an additional test for the specificity of evoked multicellular activity patterns, stimulation at two different sites within CA3 yielded clearly distinct waveforms of evoked SPW-Rs within CA1
A

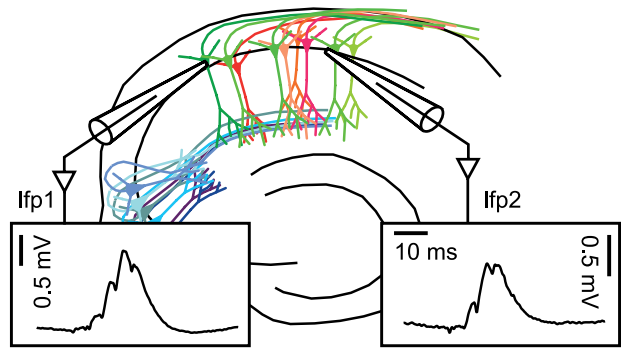

B
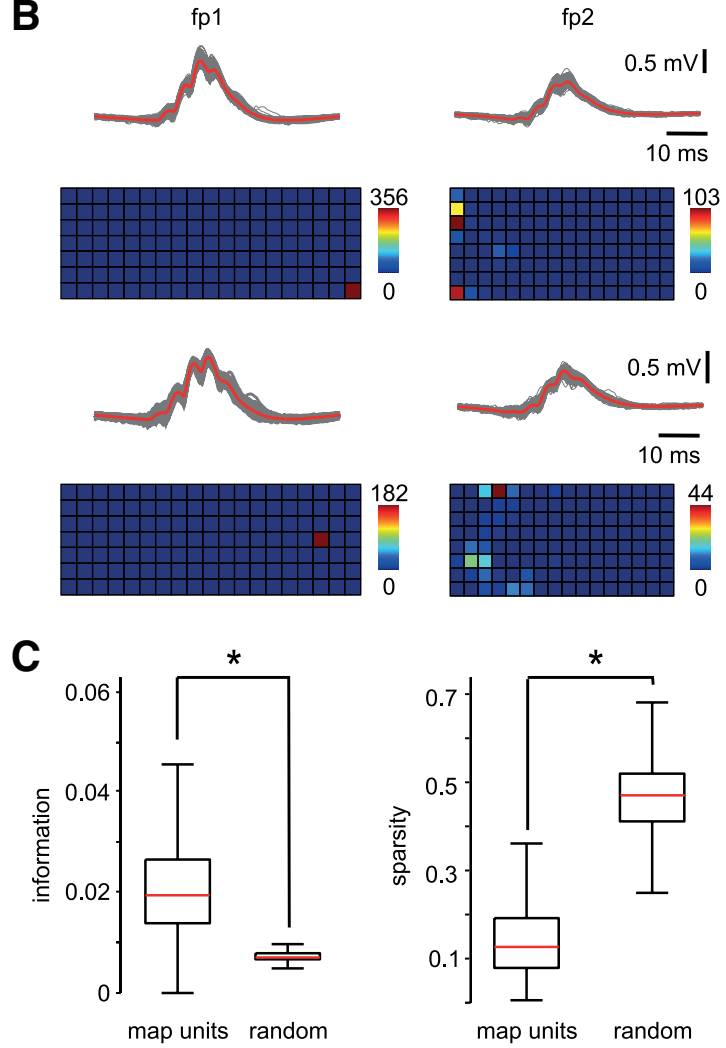

Figure 5. Modulation of SPW-R waveforms during propagation along CA1. A, Measuring field potentials at distant positions in CA1 allows analyzing the propagation of sharp waves along CA1. A given SPW-R waveform at tetrode position 1 is followed by well defined waveform patterns at tetrode 2. $\boldsymbol{B}$, Left panel, Two different classes of SPW-Rs that were recorded at position Ifp 1 shown in $\boldsymbol{A}$. The gray traces are the raw data of all events, the red traces are the means, and the hit maps show corresponding best matching SOM units. Right panel, Raw traces (gray), mean waveforms (red), and hit maps of all corresponding events at position Ifp2. Note the sparse distribution of events in the downstream hit map indicating a strong correlation between waveforms at Ifp1 and Ifp2, but also some processing of SPW-Rs during propagation. C, Information and sparsity of the concurrent SPW-R distribution at tetrode 2 are significantly different from randomly chosen SPW- $R$; ${ }^{*} p<0.05$.

(Fig. 4C; supporting information, available at www.jneurosci.org as supplemental material). Both types of evoked events mapped onto restricted, but clearly distinct areas of the SOM (Fig. 4C) $(n=8)$.

In summary, these data show that different SPW-R waveforms in CA1 result from the preceding activation of defined neuronal subpopulations within CA3. These subnetworks can be reliably activated by a rather unspecific stimulation of small regions within CA3.

\section{Modulation of SPW-R waveforms during propagation along CA1}

The propagation of sharp waves along CAl offers an independent possibility to test whether different SPW-R waveforms corre- 
A
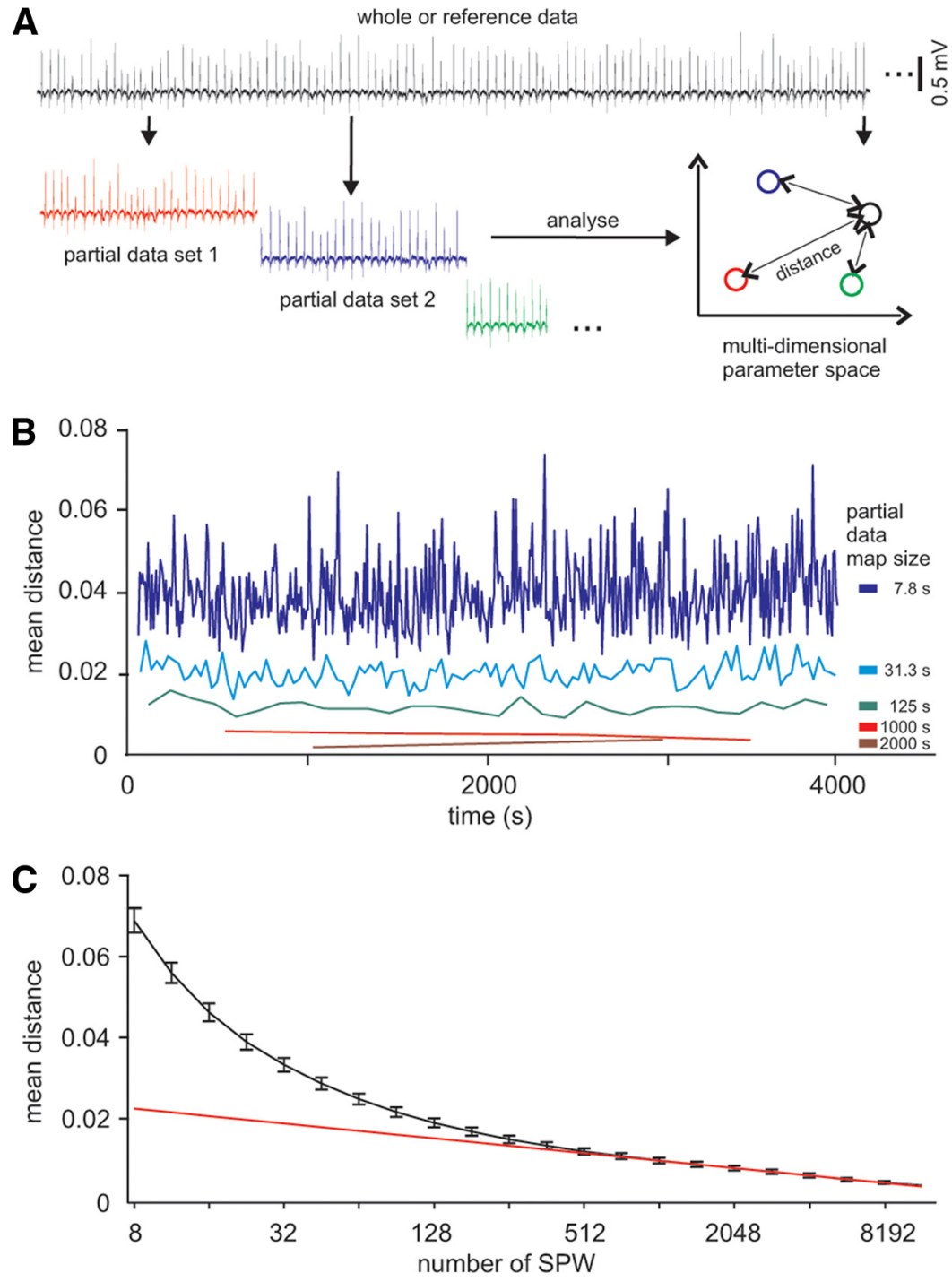

Figure 6. SPW-R waveforms are stable over time and their number is limited. $\boldsymbol{A}$, The whole data set was split into shorter segments that underwent SOM classification. Distance between resulting partial SPW-R maps and the whole data map is depicted (right) and was taken to assess waveform stability. $\boldsymbol{B}$, Distance between partial SPW-R maps depends on sample size. For each length of partial data set, however, distance between partial data maps and whole data map is stable over time. $\boldsymbol{C}$, Relationship between partial data map size and mean distance to whole data map. The sudden deviation from the logarithmic correlation at low partial data map sizes $(<500)$ indicates incomplete representation of SPW-R waveforms.

spond to different groups of coactive neurons: in this case, there should be a stable correlation between SPW-R waveforms recorded at different positions along CA1. We therefore tested the correlations between corresponding SPW-Rs recorded at two distinct sites along the CA1 pyramidal cell layer. Indeed, a given SPW-R waveform at tetrode position 1 was followed by well defined (although different) waveform patterns at tetrode 2 (Fig. $5 A, B)$. Downstream patterns were, however, slightly more diverse than the corresponding upstream waveforms. Information and sparsity of the correlating SPW-R at tetrode 2 were significantly different from randomly chosen SPW-R (Fig. 5C). Thus, multicellular activity patterns underlying SPW-R show a high degree of stability, but also some processing, during propagation along CA1.

\section{SPW-R waveforms are stable over time}

Finally, we tested the stability of recurring prototypic waveforms over time. We constructed partial maps from intervals covering varying lengths of the full recording duration ( $n=19$ experiments) (Fig. $6 A-C)$. As expected, maps from shorter time intervals showed increasing difference toward the full data map. Thus, few SPW-Rs cannot provide a full representation of all occurring waveforms (Fig. 6B; supporting information, available at www.jneurosci. org as supplemental material). Importantly, the distance between partial and full maps did not change systematically over recording time (Fig. 6B), underlining stability of SPW-R waveforms over at least $4000 \mathrm{~s}$ (runs test) (see supporting information, available at www.jneurosci.org as supplemental material). This temporal stability allowed us to use the distance parameter for assessing the quality of representation by small numbers of individual events. As expected, the mean distance between partial maps and full maps increased when fewer events were used to construct the partial maps (Fig. 6C). At values below $\sim 500$ individual events per partial map, the slope of the distance plot became increasingly steep, indicative of a lower limit of different events needed for a representation of the full spectrum of occurring waveforms.

\section{Discussion}

Field potentials reflect weighted averages of local neuronal activity. Here, we show that individual, clearly delineated network events in hippocampal slices (SPWRs) contain specific signatures of the underlying multicellular activity patterns: (1) they can be robustly classified into distinct waveforms, (2) waveforms recur stably over prolonged recording times, and (3) phase-locked discharges of single units tend to fire within a limited set of SPW-R waveforms. Therefore, field SPW-Rs are representations of defined, transiently stable groups of neurons. This close correspondence between field potentials and the underlying multicellular activity patterns may be of practical importance for the analysis of cognitive processes in humans. It also underlines the specificity of information processing in sharp wave-ripple complexes that have been implicated in declarative memory consolidation.

At first glance, the stable recurrence of defined SPW-R patterns appears surprising. Local field potentials result from current flow through cell membranes, with major contributions from subthreshold postsynaptic currents (Johnston and $\mathrm{Wu}$, 1999). Their interpretation is confounded by multiple factors, including their limited spatial extension (Cohen and Miles, 2000), laminar changes along the dendritic-axonal axis, other non-isotropic properties of the extracellular space (Bédard et al., 2004), far field effects (Nicholson, 1973; Mitzdorf, 1985), and activity-dependent changes in extracellular ion concentrations (Kann et al., 2003). Therefore, field potentials provide rather indirect reflections of the underlying network activity. Action 
potentials of one or few neurons can have significant impact on the local field, either by their direct contribution (Rasch et al., 2009) or by synaptically mediated effects on multiple target cells (Mikkonen et al., 2006; Huttunen et al., 2008). In our recordings, it is unlikely that SPW-R waveforms depended critically on the contribution of single units. Events were sorted into the same waveform class regardless of whether a coupled unit fired on an individual event or not. Moreover, the most dominant principal components for SPW-R sorting reflected sharp waves or oscillations at $\sim 200 \mathrm{~Hz}$. Both components are clearly slower than the contribution of single units.

The exact mechanisms linking SPW-R pattern and discharges of defined units are not known. One simple link may be that the amplitude of sharp waves is directly correlated with the number of recruited neurons. Therefore, we normalized the sharp waves to identical amplitudes before performing the PCA and sorting. Nevertheless, units showed almost identical values of waveform preference, indicating that unit preferences are only weakly influenced by SPW-R amplitude. Furthermore, supplemental Figure S6 (available at www.jneurosci.org as supplemental material) shows units $(8,13)$ that are clearly correlated to small-amplitude sharp waves, indicating that other features of the waveform may be equally important. Moreover, even the slight preference of units for larger sharp waves - and their absence in smaller waveform types-indicates specificity of unit firing.

The position of our recording electrode within stratum pyramidale favors the detection of perisomatic or proximal-dendritic synaptic potentials that are mainly inhibitory. Perisomatic basket cells are strongly activated during SPW-R (Klausberger et al., 2003) and are likely to contribute to the high-frequency ripple oscillation (Csicsvari et al., 1998; Klausberger et al., 2003; Hartwich et al., 2009) as well as to other oscillating states (Johnston and $\mathrm{Wu}, 1999$; Whittington and Traub, 2003; Buzsáki et al., 2004; Mann and Paulsen, 2007; Oren et al., 2010). Network bursts similar to SPW-R can indeed be influenced by the discharge of single perisomatic interneurons (Ellender et al., 2010). Although the widespread axonal arbors of perisomatic interneurons enable such strong effects at the network level, the selection of specific assembly members is most likely achieved by more selective, excitatory connections (Draguhn et al., 1998; Memmesheimer, 2010). In any case, the intricate connections between interneurons and pyramidal cells make it likely that different neuronal assemblies activate different sets of interneurons.

Our stimulation experiments show that the activation of distinct multicellular activity patterns depends mostly, but not entirely, on the upstream pattern of activity in CA3 (Both et al., 2008). A similar strong influence of few neurons in CA3 on network patterns in CA1 has previously been shown for theta and gamma oscillations in vivo (Mikkonen et al., 2006). At the same time, recordings with two spatially separated electrodes revealed degenerating waveform fidelity along CA1 (Fig. 5). This result points toward computational processes beyond mere signal propagation within CA1. It should also be kept in mind that we did not study interference from the entorhinal cortex, which is involved in signal comparison and novelty detection in CA1 (Vago and Kesner, 2008; Van Cauter et al., 2008).

Our stimulation experiments yielded a lower estimate of 10 different classes of SPW-R waveforms in CA1. It is tempting to relate this estimate to the number of distinct hippocampal assemblies (e.g., for coding of spatial memory). However, the correlation between single units and field potential waveforms does not provide a direct observation of distinct neuronal assemblies. Moreover, the hippocampal slice is a partially de-afferentiated subnetwork of the mouse hippocampus. It is, therefore, difficult to compare our number with theoretical estimates of the hippocampal storage capacity in vivo. Recent model calculations suggested 1500-100,000 different memory-representing assemblies in the recurrent network of rodent CA3 (Leibold and Kempter, 2006; de Almeida et al., 2007). Measuring and sorting different SPW-R waveforms in vivo may, in future work, provide a direct experimental approach to such numbers.

Our data may be relevant for the interpretation of electrophysiological data from animals and humans during behavioral or cognitive tasks. Indeed, high-resolution EEG or magnetoencephalographic recordings in humans have revealed event-related waveforms for different mnemonic (Fernandez et al., 1999; Fell et al., 2001) or perceptual (Fries et al., 2001; Schneider et al., 2005) processes. Similar distinctions have also been achieved during highresolution functional imaging (Norman et al., 2006). Although these authors report categorical distinctions, rigid sorting of individual waveform patterns may allow for the detection of individual, content-specific neuronal representations.

\section{References}

Bédard C, Kröger H, Destexhe A (2004) Modeling extracellular field potentials and the frequency-filtering properties of extracellular space. Biophys J 86:1829-1842.

Blanche TJ, Swindale NV (2006) Nyquist interpolation improves neuron yield in multiunit recordings. J Neurosci Methods 155:81-91.

Both M, Bähner F, von Bohlen und Halbach O, Draguhn A (2008) Propagation of specific network patterns through the mouse hippocampus. Hippocampus 18:899-908.

Bragin A, Engel J Jr, Wilson CL, Fried I, Buzsáki G (1999) High-frequency oscillations in human brain. Hippocampus 9:137-142.

Brun VH, Leutgeb S, Wu HQ, Schwarcz R, Witter MP, Moser EI, Moser MB (2008) Impaired spatial representation in CAl after lesion of direct input from entorhinal cortex. Neuron 57:290-302.

Buzsáki G (1989) Two-stage model of memory trace formation: a role for “noisy" brain states. Neuroscience 31:551-570.

Buzsáki G, Chrobak JJ (1995) Temporal structure in spatially organized neuronal ensembles: a role for interneuronal networks. Curr Opin Neurobiol 5:504-510.

Buzsáki G, Draguhn A (2004) Neuronal oscillations in cortical networks. Science 304:1926-1929.

Buzsáki G, Geisler C, Henze DA, Wang XJ (2004) Interneuron diversity series: circuit complexity and axon wiring economy of cortical interneurons. Trends Neurosci 27:186-193.

Chrobak JJ, Buzsáki G (1996) High-frequency oscillations in the output networks of the hippocampal-entorhinal axis of the freely behaving rat. J Neurosci 16:3056-3066.

Cohen I, Miles R (2000) Contributions of intrinsic and synaptic activities to the generation of neuronal discharges in in vitro hippocampus. J Physiol 524:485-502.

Csicsvari J, Hirase H, Czurko A, Buzsáki G (1998) Reliability and state dependence of pyramidal cell-interneuron synapses in the hippocampus: an ensemble approach in the behaving rat. Neuron 21:179-189.

Csicsvari J, Hirase H, Czurkó A, Mamiya A, Buzsáki G (1999) Oscillatory coupling of hippocampal pyramidal cells and interneurons in the behaving Rat. J Neurosci 19:274-287.

Csicsvari J, Hirase H, Mamiya A, Buzsáki G (2000) Ensemble patterns of hippocampal CA3-CA1 neurons during sharp wave-associated population events. Neuron 28:585-594.

de Almeida L, Idiart M, Lisman JE (2007) Memory retrieval time and memory capacity of the CA3 network: role of gamma frequency oscillations. Learn Mem 14:795-806.

Draguhn A, Traub RD, Schmitz D, Jefferys JG (1998) Electrical coupling underlies high-frequency oscillations in the hippocampus in vitro. Nature 394:189-192.

Ellender TJ, Nissen W, Colgin LL, Mann EO, Paulsen O (2010) Priming of hippocampal population bursts by individual perisomatic-targeting interneurons. J Neurosci 30:5979-5991.

Fell J, Klaver P, Lehnertz K, Grunwald T, Schaller C, Elger CE, Fernández G 
(2001) Human memory formation is accompanied by rhinal-hippocampal coupling and decoupling. Nat Neurosci 4:1259-1264.

Fernández G, Effern A, Grunwald T, Pezer N, Lehnertz K, Dümpelmann M, Van Roost D, Elger CE (1999) Real-time tracking of memory formation in the human rhinal cortex and hippocampus. Science 285:1582-1585.

Foster DJ, Wilson MA (2006) Reverse replay of behavioural sequences in hippocampal place cells during the awake state. Nature 440:680-683.

Frank LM, Stanley GB, Brown EN (2004) Hippocampal plasticity across multiple days of exposure to novel environments. J Neurosci 24:7681-7689.

Fries P, Neuenschwander S, Engel AK, Goebel R, Singer W (2001) Rapid feature selective neuronal synchronization through correlated latency shifting. Nat Neurosci 4:194-200.

Harris KD, Henze DA, Csicsvari J, Hirase H, Buzsáki G (2000) Accuracy of tetrode spike separation as determined by simultaneous intracellular and extracellular measurements. J Neurophysiol 84:401-414.

Hartwich K, Pollak T, Klausberger T (2009) Distinct firing patterns of identified basket and dendrite-targeting interneurons in the prefrontal cortex during hippocampal theta and local spindle oscillations. J Neurosci 29:9563-9574.

Hazan L, Zugaro M, Buzsáki G (2006) Klusters, NeuroScope, NDManager: a free software suite for neurophysiological data processing and visualization. J Neurosci Methods 155:207-216.

Hebb DO (1949) The organization of behavior: a neurophysiological theory. New York: Wiley.

Huttunen JK, Gröhn O, Penttonen M (2008) Coupling between simultaneously recorded BOLD response and neuronal activity in the rat somatosensory cortex. Neuroimage 39:775-785.

Johnston D, Wu SM (1999) Foundations of cellular neurophysiology. Cambridge, MA: MIT.

Kann O, Schuchmann S, Buchheim K, Heinemann U (2003) Coupling of neuronal activity and mitochondrial metabolism as revealed by $\mathrm{NAD}(\mathrm{P}) \mathrm{H}$ fluorescence signals in organotypic hippocampal slice cultures of the rat. Neuroscience 119:87-100.

Klausberger T, Somogyi P (2008) Neuronal diversity and temporal dynamics: the unity of hippocampal circuit operations. Science 321:53-57.

Klausberger T, Magill PJ, Márton LF, Roberts JD, Cobden PM, Buzsáki G, Somogyi P (2003) Brain-state- and cell-type-specific firing of hippocampal interneurons in vivo. Nature 421:844-848.

Kohonen T (1995) Self-organizing maps. Berlin: Springer.

Leibold C, Kempter R (2006) Memory capacity for sequences in a recurrent network with biological constraints. Neural Comput 18:904-941.

Leutgeb JK, Leutgeb S, Treves A, Meyer R, Barnes CA, McNaughton BL, Moser MB, Moser EI (2005) Progressive transformation of hippocampal neuronal representations in "morphed" environments. Neuron 48:345-358.

Lisman JE (1999) Relating hippocampal circuitry to function: recall of memory sequences by reciprocal dentate-CA3 interactions. Neuron 22:233-242.

Maier N, Güldenagel M, Söhl G, Siegmund H, Willecke K, Draguhn A (2002) Reduction of high-frequency network oscillations (ripples) and pathological network discharges in hippocampal slices from connexin 36-deficient mice. J Physiol 541:521-528.

Maier N, Nimmrich V, Draguhn A (2003) Cellular and network mechanisms underlying spontaneous sharp wave-ripple complexes in mouse hippocampal slices. J Physiol 550:873-887.
Mann EO, Paulsen O (2007) Role of GABAergic inhibition in hippocampal network oscillations. Trends Neurosci 30:343-349.

Memmesheimer RM (2010) Quantitative prediction of intermittent highfrequency oscillations in neural networks with supralinear dendritic interactions. Proc Natl Acad Sci U S A 107:11092-11097.

Mikkonen JE, Huttunen J, Penttonen M (2006) Contribution of a single CA3 neuron to network synchrony. Neuroimage 31:1222-1227.

Mitzdorf U (1985) Current source-density method and application in cat cerebral cortex: investigation of evoked potentials and EEG phenomena. Physiol Rev 65:37-100.

Nádasdy Z, Hirase H, Czurkó A, Csicsvari J, Buzsáki G (1999) Replay and time compression of recurring spike sequences in the hippocampus. J Neurosci 19:9497-9507.

Nicholson C (1973) Theoretical analysis of field potentials in anisotropic ensembles of neuronal elements. IEEE Trans Biomed Eng 20:278-288.

Norman KA, Polyn SM, Detre GJ, Haxby JV (2006) Beyond mind-reading: multi-voxel pattern analysis of fMRI data. Trends Cogn Sci 10:424-430.

O’Keefe J, Dostrovsky J (1971) The hippocampus as a spatial map. Preliminary evidence from unit activity in the freely-moving rat. Brain Res 34:171-175.

O'Keefe J, Nadel L (1978) The hippocampus as a cognitive map. Oxford: Oxford UP

O’Keefe J, Recce ML (1993) Phase relationship between hippocampal place units and the EEG theta rhythm. Hippocampus 3:317-330.

Oren I, Hájos N, Paulsen O (2010) Identification of the current generator underlying cholinergically induced gamma frequency field potential oscillations in the hippocampal CA3 region. J Physiol 588:785-797.

Rasch M, Logothetis NK, Kreiman G (2009) From neurons to circuits: linear estimation of local field potentials. J Neurosci 29:13785-13796.

Schmitzer-Torbert N, Jackson J, Henze D, Harris K, Redish AD (2005) Quantitative measures of cluster quality for use in extracellular recordings. Neuroscience 131:1-11.

Schneider P, Sluming V, Roberts N, Bleeck S, Rupp A (2005) Structural, functional, and perceptual differences in Heschl's gyrus and musical instrument preference. Ann N Y Acad Sci 1060:387-394.

Skaggs WE, McNaughton BL, Gothart KM, Markus EJ (1993) Advances in neural information processing systems. San Mateo, CA: Morgan Kaufmann.

Treves A, Rolls ET (1994) Computational analysis of the role of the hippocampus in memory. Hippocampus 4:374-391.

Vago DR, Kesner RP (2008) Disruption of the direct perforant path input to the CA1 subregion of the dorsal hippocampus interferes with spatial working memory and novelty detection. Behav Brain Res 189:273-283.

Van Cauter T, Poucet B, Save E (2008) Delay-dependent involvement of the rat entorhinal cortex in habituation to a novel environment. Neurobiol Learn Mem 90:192-199.

Whittington MA, Traub RD (2003) Interneuron diversity series: inhibitory interneurons and network oscillations in vitro. Trends Neurosci 26:676-682.

Wilson MA, McNaughton BL (1993) Dynamics of the hippocampal ensemble code for space. Science 261:1055-1058.

Wilson MA, McNaughton BL (1994) Reactivation of hippocampal ensemble memories during sleep. Science 265:676-679.

Ylinen A, Bragin A, Nádasdy Z, Jandó G, Szabó I, Sik A, Buzsáki G (1995) Sharp wave-associated high-frequency oscillation $(200 \mathrm{~Hz})$ in the intact hippocampus: network and intracellular mechanisms. J Neurosci 15:30-46. 